\title{
Surface water waves and tsunamis
}

\author{
By Walter Craig \\ Department of Mathematics and Statistics \\ McMaster University \\ Hamilton, Ontario $L 8 S 4 \mathrm{~K} 1$, Canada \\ http://www.math.mcmaster.ca/ craig
}

Because of the enormous earthquake in Sumatra on December 26, 2004, and the devastating tsunami which followed, I have chosen the focus of my mini-course lectures at this year's PASI to be on two topics which involve the dynamics of surface water waves. These topics are of interest to mathematicians interested in wave propagation, and particularly to Chilean scientists, I believe, because of Chile's presence on the tectonically active Pacific Rim.

My first lecture will describe the equations of fluid dynamics for the free surface above a body of fluid (the ocean surface), and the linearized equations of motion. From this we can predict the travel time of the recent tsunami from its epicenter off of the north Sumatra coast to the coast of nearby Thailand, the easy coasts of Sri Lanka and south India, and to Africa. In fact the signal given by ocean waves generated by the Sumatra earthquake was felt globally; within 48 hours distinguishable tsunami waves were measured by wave gages in Antarctica, Chile, Rio di Janeiro, the west coast of Mexico, the east coast of the United States, and at Halifax, Nova Scotia.

To describe ocean waves we will formulate the full nonlinear fluid dynamical equations as a Hamiltonian system (Zakharov 1968 [19]), and we will introduce the Greens function and the Dirichlet-Neumann operator for the fluid domain along with the harmonic analysis of the theory of their regularity. From an asymptotic theory of scaling transformations we will derive the known Boussinesq-like systems and the $\mathrm{KdV}$ and KP equations which govern the asymptotic behavior of tsunami waves over an idealized flat bottom. When the bottom is no longer assumed to be perfectly flat, a related theory (Rosales \& Papanicolaou 1983 [13]) (Craig, Guyenne, Nicholls \& Sulem 2005 [6]) gives a family of model equations taking this into account.

My second lecture will describe a series of recent results in PDE, numerical results, and experimental results on the nonlinear interactions of solitary surface water waves. In contrast with the case of the $\mathrm{KdV}$ equations (and certain other integrable PDE), the Euler equations for a free surface do not admit clean ('elastic') interactions between solitary wave solutions. This has been a classical concern of oceanographers for several decades, but only recently have there been sufficiently accurate and thorough numerical simulations which quantify the degree to which solitary waves lose energy during interactions (Cooker, Wiedman \& Bale 1997 [3]) (Craig, Guyenne, Hammack, Henderson \& Sulem 2006 [4]). It is striking that this degree of 'inelasticity' is remarkably small. I'll describe this work, as well as recent results on the initial value problem which are very relevant to this phenomenon (Schneider \& Wayne 2000 [14]) (Wright 2005 [18]). 
Keywords: Tsunamis, nonlinear surface water waves

\section{Tsunamis and ocean waves}

The name 'tsunami' in Japanese originally means 'harbor wave', but the word has come to mean a large and potentially very powerful series of ocean waves which are occasionally generated by movements of the ocean floor. Very infrequently these can be extremely large and very dangerous, and can have a destructive impact on coastal areas of the world's oceans. Small tsunamis are generated relatively often, most commonly by tectonic activity, but sometimes by other means as well, such as underwater landslides. Serious tsunamis, to the point of loss of life and major coastal destruction, take place on the order of once every several decades. I think we were all personally touched by the deadly Boxing Day Tsunami in 2004 which was generated by the major earthquake in the Sunda Trench subduction zone off the west coast of the island of Sumatra. It is a challenge for mathematicians with interests in the hydrodynamics of ocean waves to seek a credible rôle to play in predicting and/or articulating the danger of a tsunami or in alleviating the force of their impact.

Taking a naïve point of view, the most effective means to predict major tsunamis is to predict major earthquakes. However this an ambitious objective to say the least, and despite the research of many decades of earth scientists this remains a grand challenge problem, on which major progress will take much time. The question remains, however, as to find credible aspects of tsunami prediction on which mathematicians can contribute. It seems to me that there are many important open questions in the modeling of (1) tsunami wave generation in the event of a major earthquake, (2) wave propagation across the opean ocean, and (3) wave impact upon the coastlines affected by the event. Indeed the design of tsunami early warning systems or some of its components involves mathematical modeling of solutions of the partial differential equations describing ocean wave dynamics, and computer simulation of solutions which, if they are to be an effective warning, must be performed in faster than real time. It is also an important problem to be able to clarify the character of tsunami waves, in particular those features of the waves as they impact on coastal areas which can effect tsunami safety codes in engineering and architecture.

In my first talk on tsunamis, I plan to discuss some of the elementary features of the phenomenon, most of which are essentially well know facts in the theory of surface water waves. Some however are new, or at least reflect a modern viewpoint. For the most part I will place these in the context of the Boxing Day Tsunami 2004, which is clearest in our memory, in order to bring perspective to this event. The basic details are that this tsunami was generated by a major earthquake that took place along the Sunda Trench, which is the subduction zone along of the west coast of the island of Sumatra. The earthquake took place on 26 December 2004 at $7 \mathrm{~h} 58$ local time, and its epicenter was located approximately $250 \mathrm{~km}$ off shore. It was initially classified as a magnitude $M_{w}=8.5 \sim 9$, but after subsequent analysis (which took several weeks) the estimates of its strength were upgraded to $M_{w}=9.3$. The earthquake caused a rupture of the ocean floor moving essentially to the north-northwest along the subduction zone, of length approximately $1200 \mathrm{~km}$. 


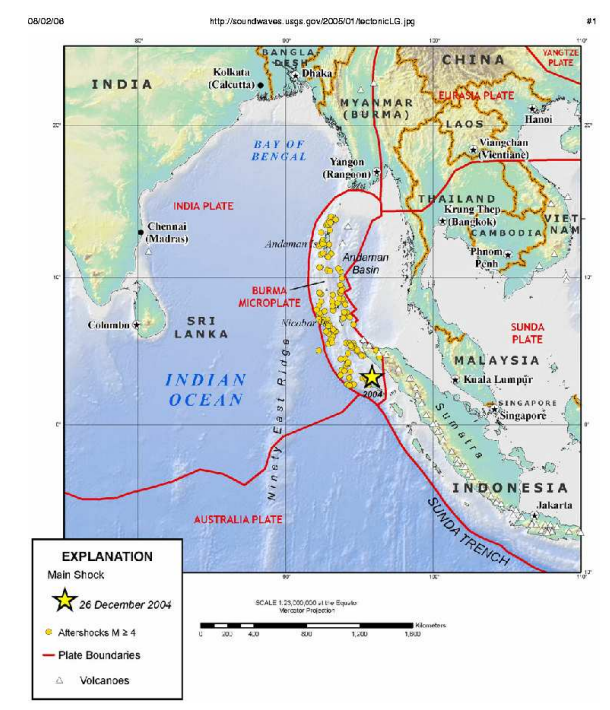

Figure 1. Northeastern Indian Ocean tectonic setting. USGS website: http://soundwaves.usgs.gov/2005/01/tectonicLG.jpg

The magnitude of the horizontal slip along this rupture is estimated as being $21 \mathrm{~m}$ in the southern parts, to $15 \mathrm{~m}$ in more northern locations. Vertical displacement, which is perhaps more relevant to tsunami generation, is estimated to be between $2 m$ to $10 m$. It was an unusual earthquake in the eyes of seismologists, as the rupture moved relatively slowly, acting somewhat like a zipper from the southeastern to the northwestern part of the rupture, and lasting between 15 to 20 minutes in all. A diagram of the northeastern Indian Ocean tectonic region gives a picture of the setting of this earthquake and allows us to put the event into perspective (Figure 1).

The slip along such an extended region acted as a line source generating tsunami waves, which propagated both through the Indian Ocean to the west, and the Andaman Basin to the east. Soon after being generated, devastating tsunami waves hit the coast of Sumatra itself and cause extensive damage and loss of life. The tsunami waves traveled at approximately $360 \mathrm{~km} / \mathrm{hr}$ eastwards through the Andaman Basin, to impact upon the west coast of the Malay Peninsula, including portions of Thailand and Myanmar, about one hour after the earthquake. Waves traveling at approximately $720 \mathrm{~km} / \mathrm{hr}$ propagated west through the lower Bay of Bengal / Indian Ocean, impacting on Sri Lanka and the east coast of southern India approximately 2 hours after the earthquake. The tsunami was in fact felt globally; within 7 hours on the east African coast, within 20 hours on the South Atlantic Ocean in Rio de Janeiro, within 23 hours on the South Pacific Ocean in Chile, and in 29 hours in Halifax, Nova Scotia. A numerical model of the global propagation of the Boxing Day Tsunami was published in Science Express on August 25 2005, and in [17], (Figure 2) which shows this effect. 


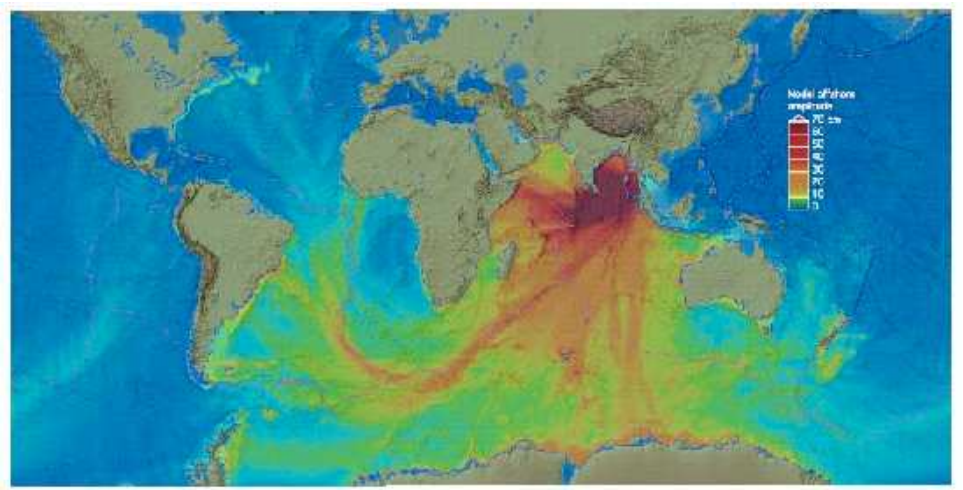

Figure 2. The global reach of the 26 December 2004 Sumatra tsunami: Science Express on 25 August 2005, Science 23 September 2005, Vol. 309. no. 5743, pp. 2045 - 2048 Vasily Titov, Alexander B. Rabinovich, Harold O. Mofjeld, Richard E. Thomson, Frank I. Gonzàlez

\section{Equations of motion for potential flow}

Oceanographers generally treat ocean waves as free surface waves in an ideal fluid. This is to say that one works with potential flow, for which the velocity of the fluid in Eulerian coördinates is given in terms of a velocity potential $\varphi$, such that

$$
\Delta \varphi=0
$$

in the domain occupied by the fluid. This fluid domain is bounded below by $\{y=$ $\left.-h(x), x \in \mathbb{R}^{n-1}\right\}$, while the free surface is given in the form of a graph $\{y=$ $\eta(x, t)\}$. For tsunami propagation we should really be treating ocean waves on a sphere or on certain subsets of the sphere corresponding to ocean regions. However for reasons of simplicity we will simply consider the fluid domain to be $x \in \mathbb{R}^{2}$, $-h(x)<y<\eta(x, t)$. On the bottom boundary of the fluid domain we impose

$$
N \cdot \nabla \varphi=0 \quad \text { on } \quad y=-h(x),
$$

and on the free surface we impose the two classical boundary conditions

$$
\left.\begin{array}{r}
\partial_{t} \varphi+\frac{1}{2}|\nabla \varphi|^{2}+g \eta=0 \\
\partial_{t} \eta+\partial_{x} \eta \cdot \partial_{x} \varphi-\partial_{y} \varphi=0
\end{array}\right\} \quad \text { on } \quad y=\eta(x, t)
$$

This will describe the motion of ocean waves in the setting in which the bottom is fixed, which is in our case to be during the second phase of wave propagation of the tsunami after the time at which they have been generated. The boundary conditions describing the generation of tsunami waves are different, as the bottom boundary can no longer assumed to be stationary. In particular the sea bottom should be given as $\{y=-h(x, t)\}$ and one must replace the boundary conditions (2.2) by the following expression

$$
N \cdot \nabla \varphi=\partial_{t} h(x, t) \quad \text { on } \quad y=-h(x, t),
$$

where the outward normal to the bottom boundary is given by $N=\left(-\partial_{x} h,-1\right)$. In studies of the generation of a tsunami from a given source, these are appropriate boundery conditions to work with. 
These equations can be posed in the form of a Hamilton system, a fact which is due to V.E. Zakharov [19]. Zakharov's Hamiltonian can be rewritten [7] as

$$
H(\eta, \xi)=\frac{1}{2} \int_{\mathbb{R}^{n-1}} \xi G(\eta, h) \xi+g \eta^{2} d x .
$$

In this expression we write $\xi(x):=\varphi(x, \eta(x))$, and the Dirichlet integral, which represents the kinetic energy, is expressed in terms of the Dirichlet - Neumann operator

$$
G(\eta, h) \xi:=\left.\sqrt{1+\left|\partial_{x} \eta\right|^{2}} N \cdot \nabla \varphi\right|_{y=\eta},
$$

where the harmonic extension $\varphi$ of the top boundary conditions $\xi(x)$ to the fluid domain is required to satisfy the bottom boundary conditions (2.4). The equations (2.1) through (2.3) are written in the canonical form

$$
\partial_{t}\left(\begin{array}{l}
\eta \\
\xi
\end{array}\right)=\left(\begin{array}{cc}
0 & 1 \\
-1 & 0
\end{array}\right)\left(\begin{array}{l}
\delta_{\eta} H \\
\delta_{\xi} H
\end{array}\right) .
$$

Explicitely, Hamilton's canonical equations (2.7) have the form

$$
\begin{aligned}
\partial_{t} \eta=G(\eta, h) \xi & \\
\partial_{t} \xi=-g \eta & +\frac{-1}{2\left(1+\left|\partial_{x} \eta\right|^{2}\right)}\left[\left(\partial_{x} \xi\right)^{2}-(G(\eta, h) \xi)^{2}-2 \partial_{x} \eta \partial_{x} \xi G(\eta, h) \xi\right. \\
& \left.\quad+\left(\left|\nabla_{x} \xi\right|^{2}\left|\nabla_{x} \eta\right|^{2}-\left(\nabla_{x} \eta \cdot \nabla_{x} \xi\right)^{2}\right)\right]
\end{aligned}
$$

One of the terms of the second component (2.10) of this Hamiltonian vector field has two fewer terms in the case of the water wave equations in two dimensions.

The time evolution of (2.7) conserves a number of physical quantities in addition to the Hamiltonian, including the added mass

$$
M(\eta)=\int_{\mathbb{R}^{n-1}} \eta(x, t) d x .
$$

Conservation of this quantity is verified by the following identity

$$
\{M, H\}=0,
$$

where the Poisson bracket between two functionals $F$ and $H$ is given by

$$
\{F, H\}=\int_{\mathbb{R}^{n-1}} \delta_{\eta} F \delta_{\xi} H-\delta_{\xi} F \delta_{\eta} H d x .
$$

The momentum, or impulse of a solution is another conserved quantity in the case that the bottom boundary of the fluid region is flat, $\partial_{x} h=0$;

$$
I(\eta, \xi)=\int_{\mathbb{R}^{n-1}} \eta(x, t) \partial_{x} \xi(x, t) d x,
$$

and we can verify by the calculation that

$$
\{I, H\}=0 .
$$


In this case the center of mass of a solution is given by the expression

$$
C(\eta)=\int_{-\infty}^{\infty} x \eta(x, t) d x
$$

and it evolves linearly in time; indeed its time derivative is a constant of motion

$$
\begin{aligned}
\frac{d}{d t} C & =\int_{\mathbb{R}^{n-1}} x \partial_{t} \eta(x, t) d x=\int_{\mathbb{R}^{n-1}} x G(\eta) \xi d x \\
& =\int_{\mathbb{R}^{n-1}} \xi G(\eta) x d x=\int_{\mathbb{R}^{n-1}} \xi\left(-\partial_{x} \eta\right) d x=I(\eta, \xi) .
\end{aligned}
$$

\section{Linear wave speed}

The equations (2.8) do not have the property of finite propagation speed, as does the classical wave equation, since the requirement of incompressibility for potential flow involves global information about the fluid. But solutions do have an effective propagation speed, which is finite for fluid regions which are not infinitely deep. Although we would like to invoke this principle in an ocean basin as complicated as the Indian Ocean, it is easier to derive it in the case that the bottom is flat, and the deformations of the free surface are sufficiently small to be treated by linear theory. The fluid region is therefore $\{(x, y):-h<y<0\}$, and the linearized equations of motion are given by the quadratic part of the Hamiltonian

$$
H^{(2)}=\frac{1}{2} \int_{\mathbb{R}^{n-1}} \xi G(0, h) \xi+g \eta^{2} d x .
$$

The Dirichlet - Neumann operator $G(0, h)$ for a strip is given in terms of a Fourier multiplier operator, which can be derived by solving the boundary value problem for the velocity potential

$$
\begin{aligned}
\Delta \varphi & =0 \\
\varphi(x, 0) & =\xi(x) \\
-\partial_{y} \varphi(x,-h) & =0
\end{aligned}
$$

Then the operator $G$ is defined by the expression $G(0, h) \xi(x):=\partial_{y} \varphi(x, 0)$. When the particular boundary data $\xi(x)=e^{i k \cdot x}$ is given, the solution of the potential problem is

$$
\varphi(x, y)=\frac{\cosh (k(y+h))}{\cosh (k h)} e^{i k \cdot x},
$$

from which we deduce that

$$
G(0, h) e^{i k \cdot x}=\partial_{y} \varphi(x, 0)=\left.\frac{k \sinh (k(y+h))}{\cosh (k h)}\right|_{y=0} e^{i k \cdot x}=k \tanh (k h) e^{i k \cdot x} .
$$

The general solution is therefore given in terms of the Fourier transform of the Dirichlet data on the linearized free surface,

$$
\begin{aligned}
G(0, h) \xi(x) & =\frac{1}{(\sqrt{2 \pi})^{n-1}} \int_{\mathbb{R}^{n-1}} e^{i k \cdot x} k \tanh (h k) \hat{\xi}(k) d k \\
& =D \tanh (h D) \xi(x)
\end{aligned}
$$


where we use the notation $D=-i \partial_{x}$. Using this information we derive the dispersion relation for the linearized equations of motion, which is the frequency of the normal modes for the quadratic Hamiltonian $H^{(2)}$. Namely, the linear equations of evolution are

$$
\begin{aligned}
\partial_{t}\left(\begin{array}{l}
\eta \\
\xi
\end{array}\right) & =\left(\begin{array}{cc}
0 & 1 \\
-1 & 0
\end{array}\right)\left(\begin{array}{l}
\delta_{\eta} H^{(2)} \\
\delta_{\xi} H^{(2)}
\end{array}\right) \\
& =\left(\begin{array}{cc}
0 & D \tanh (h D) \\
-g & 0
\end{array}\right)\left(\begin{array}{l}
\eta \\
\xi
\end{array}\right) .
\end{aligned}
$$

The Fourier integral expression for the solution of this system of equations is

$$
\begin{aligned}
\left(\begin{array}{l}
\eta(x, t) \\
\xi(x, t)
\end{array}\right) & =\frac{1}{(2 \pi)^{(n-1) / 2}} \int_{\mathbb{R}^{n-1}} e^{i k \cdot x} \times \\
& \times\left(\begin{array}{cc}
\cos (\omega(k) t) & \sqrt{\frac{k \tanh (h k)}{g}} \sin (\omega(k) t) \\
-\sqrt{\frac{g}{k \tanh (h k)}} \sin (\omega(k) t) & \cos (\omega(k) t)
\end{array}\right)\left(\begin{array}{l}
\hat{\eta}_{0}(k) \\
\hat{\xi_{0}}(k)
\end{array}\right) d k
\end{aligned}
$$

where the frequency at wave number $k$ is given by the dispersion relation

$$
\omega(k)=\sqrt{g k \tanh (h k)} .
$$

With this dispersion relation, the phase velocity of solutions of (3.6) is

$$
c_{p}(k)=\frac{\omega(k)}{|k|^{2}} k,
$$

and the group velocity is

$$
c_{g}(k)=\partial_{k} \omega(k) \text {. }
$$

The significance of these two quantities is a basic fact of the theory of linear wave propagation. Each Fourier mode $e^{i k \cdot x}$ component of the initial data $\left(\eta_{0}(x), \xi_{0}(x)\right)^{T}$ propagates as governed by the expression (3.7), with phase

$$
k \cdot x-\omega(k) t=k \cdot\left(x-t \frac{\omega(k)}{|k|^{2}} k\right) .
$$

Thus for each mode, points of constant phase propagate with the phase velocity $c_{p}$. The significance of the group velocity is a bit more subtle, and involves the evolution of a wave packet. For simplicity, assume that the initial data has a smooth and compactly supported Fourier transform $\left(\hat{\eta_{0}}(k), \hat{\xi_{0}}(k)\right)^{T} \in C_{0}^{\infty}$. If this is not the case, one could decompose more general initial data using a partition of unity on first the spatial and then the Fourier transform side, after which each component would satisfy these conditions. Individual terms in the Fourier integral expression (3.7) take the form

$$
\psi(x, t)=\int_{\mathbb{R}^{n-1}} e^{i k \cdot x-i \omega(k) t} \hat{\psi}_{0}(k) d k .
$$

Consider points $(x, t)$ in space-time such that for all $k \in \operatorname{supp} \hat{\psi}_{0}$, we have

$$
\left|\frac{x}{t}-\partial_{k} \omega(k)\right|>R
$$




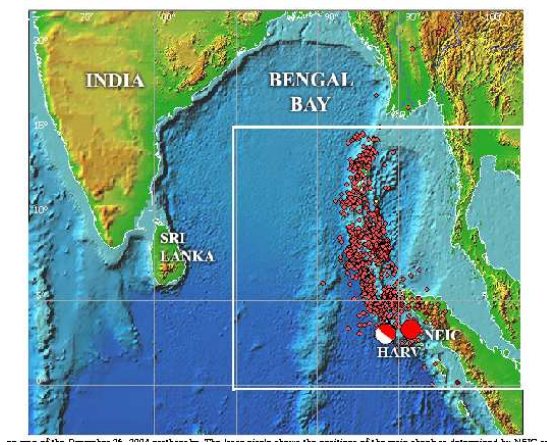

Figure 3. Novosibirsk Tsunami Laboratory website: http://tsun.sscc.ru/tsulab/20041226.htm

Then we can use the identity

$$
e^{i(k \cdot x-\omega(k) t)}=\frac{x-\partial_{k} \omega(k) t}{\left|x-\partial_{k} \omega(k) t\right|^{2}}\left(-i \partial_{k}\right) e^{i(k \cdot x-\omega(k) t)}
$$

in the integral (3.9), and because of (3.10) we can integrate by parts, to write

$$
\begin{aligned}
\psi(x, t) & =\int_{\mathbb{R}^{n-1}} e^{i(k \cdot x-\omega(k) t)}\left(i \partial_{k} \cdot\left(\frac{x-\partial_{k} \omega(k) t}{\left|x-\partial_{k} \omega(k) t\right|^{2}}\right)\right)^{N} \hat{\psi}_{0}(k) d k \\
& \leq \mathcal{O}\left((R t)^{-N}\right)
\end{aligned}
$$

for all $N$. Thus initial data organized in a wave packet with wave numbers close to $k$ do not travel very much into regions of space-time which satisfy (3.10). That is, they essentially propagate with the group velocity $\partial_{k} \omega(k)$.

In the case of the dispersion relation for water waves (3.8), the phase and group velocities tend as $|k| \rightarrow \infty$ to

$$
c_{p}(k) \sim \sqrt{g /|k|}, \quad c_{g}(k) \sim \frac{1}{2} \sqrt{g /|k|}
$$

from which we learn that short waves travel slowly in the ocean surface. We may also extrapolate that the individual wave crests within an evolving wavepacket will not be stationary, and will in fact travel somewhat faster than the wave packet itself (up to two times as fast for large wavenumber). On the other hand, as $|k| \rightarrow 0$, both the phase and group velocities tend to a constant magnitude

$$
c_{p}(k) \sim \sqrt{g h}, \quad c_{g}(k) \sim \sqrt{g h} .
$$

We will assume that large scale tsunami waves are a very low wave number phenomenon, for which the asymptotic formulae (3.12) are valid.

The primary conclusion to discuss is the speed of propagation of tsunami waves in ocean basins such as the ones in which the Boxing Day tsunami occurred. Our analysis will be based on a knowledge of the bathymetry of these oceans, which is reviewed in Figure 3 posted by the Novosibirsk Tsunami Laboratory. The distance from the epicenter of the Sumatra earthquake to the affected coast of Sri Lanka is 
about 1,550km, and the Bay of Bengal/Indian Ocean through which the tsunami waves traveled is a deep ocean, with depth of roughly $4 \mathrm{~km}$. Making the very rough approximation that $h=4 \mathrm{~km}$ and taking $g=9.8 \mathrm{~m} / \mathrm{sec}^{2}$, we can approximate the speed of the tsunami over this distance to be

$$
c=\sqrt{g h} \sim \sqrt{\left(9.8 \mathrm{~m} / \mathrm{sec}^{2}\right) \times\left(4 \times 10^{3} \mathrm{~m}\right)} \sim 2 \times 10^{2} \mathrm{~m} / \mathrm{sec}=720 \mathrm{~km} / \mathrm{hr} .
$$

Thus the approximation to the travel time for this process is 2.2 hours, which is actually about right. Similarly, the distance from the epicenter to the western coast of the Malay Peninsula, which is where the principal Thai resorts were hit, and which has coastal areas of Indonesia and Myanmar, is about $700 \mathrm{~km}$. However the tsunami waves generated in this direction propagated through the Andaman Basin, which is a shallower sea of quite variable depth. For purposes of our illustrative calculations let us take the approximate depth to be $1 \mathrm{~km}$ which is not too far off. Then we find

$$
c=\sqrt{g h} \sim \sqrt{\left(9.8 \mathrm{~m} / \mathrm{sec}^{2}\right) \times 10^{3} \mathrm{~m}} \sim 10^{2} \mathrm{~m} / \mathrm{sec}=360 \mathrm{~km} / \mathrm{hour},
$$

resulting in a travel time of approximately 1.9 hours from the initiation at the epicenter to the impact on the coast. In any case, we see that tsunami waves can carry energy very rapidly from one coastal region to another, spanning the largest distance scales of our planet in times from hours to a little more than one day.

\section{Coherent wavetrains}

When a sufficiently strong earthquake occurs, it may be felt without instruments almost instantly in a neighboring region, and when a large one occurs, it can be destructive over a large area. However even very big earthquakes do not directly cause significant damage at great distances from their source. That is, disturbances in the earth's crust travel very rapidly, but as well the largest component of their energy is dissipated after having propagated a relatively short distance from their epicenter. The fact is that tsunami waves in the ocean behave differently than this. The nonlinear character of the Euler equations for the propagation of ocean waves gives rise to the possibility that tsunami waves can propagate in coherent wave packets, with little loss of amplitude over very long distances.

We can deduce some aspects of the character of these nonlinear waves just from the starting point of several of the reports of witnesses of the impact of the Boxing Day Tsunami. Soon after the event there were a number of descriptions of peoples' experiences posted on the web. Certain of the basic facts seemed to be common to many of these; that there were several wave crests observed, 6 or 7 in the case of the Thai coast for example, and that the period between the arrival of these crests was between 15 and 45 minutes. Furthermore, measured wave amplitudes at sea did not exceed 1 to 2 meters, although on the coastline the tsunami in many places underwent an amplification to 3 up to maybe 7 meters. This latter data is not quantified with precise observational data in most cases, apparently because not many wave gages were in place or operational in the region at the time of the event. In any case, from the basic information above we can deduce some nontrivial facts about this tsunami's waveform.

For the purpose of a sample calculation, let us take a temporal period for arrival of wave crests to be $T_{0}=15 \mathrm{~min}$. The spatial period of the individual crests we can 
denote by $X_{0}$, so that the basic wavenumber of the tsunami wave is $K_{0}=2 \pi / X_{0}$. The phase velocity, which we argued above is the velocity of propagation of the individual crests, is

$$
c_{p}\left(K_{0}\right)=\sqrt{\frac{g \tanh \left(h K_{0}\right)}{K_{0}}}
$$

and the relation between the spatial and temporal periods is of course

$$
c_{p}\left(K_{0}\right) T_{0}=X_{0}=\frac{2 \pi}{K_{0}} .
$$

That is,

$$
g K_{0} \tanh \left(h K_{0}\right) T_{0}^{2}=(2 \pi)^{2} .
$$

Because we seek a small wavenumber $K_{0}$ (and because $15 \mathrm{~min}$ is $900 \mathrm{sec}$ ),

$$
(2 \pi)^{2} \sim g h K_{0}^{2} T_{0}^{2} \sim 10 \mathrm{~m} / \mathrm{sec}^{2} \times\left(4 \times 10^{3} \mathrm{~m}\right) \times(900 \mathrm{sec})^{2} \times K_{0}^{2} .
$$

Hence we find that

$$
K_{0} \sim \frac{2 \pi}{18 \times 10^{4} \mathrm{~m}} \sim 4 \times 10^{5} \mathrm{~m}^{-1},
$$

which corresponds to a spatial wavelength of $X_{0} \sim 180 \mathrm{~km}$. The slope in mid-ocean of waves of this form is approximately

$$
\left\|\partial_{x} \eta\right\|_{\infty} \sim \frac{2}{X_{0}}\|\eta\|_{\infty} \sim \frac{2 m}{18 \times 10^{4} m} \sim 10^{-5}:=\varepsilon,
$$

where applied mathematicians often think of $\varepsilon$ as a small dimensionless parameter which would be useful in a perturbation analysis of the problem. Actually in these notes I'd like to a bit more careful, and introduce two small parameters:

$$
\alpha:=\frac{a}{h}, \quad \beta:=\frac{h}{\ell},
$$

where $a$ is the amplitude of the solutions we are studying, $h$ is the fluid basin depth as before, and $\ell$ is some measure of the characteristic wavelength of our solutions. The appropriate model equations for the regime of wave propagation that we are to study are traditionally determined by the relationship between $\alpha$ and $\beta$; in case $\alpha \sim \beta^{2} \ll 1$ this is the dispersive nonlinear regime, while $\alpha \sim \beta \ll 1$ indicates the weakly nonlinear shallow water regime.

It is instructive to compare the parameters we have just deduced for the 2004 Boxing Day Tsunami with thees criteria. In the basin of the Indian Ocean/Bay of Bengal, we use the values of amplitude $a=1 \mathrm{~m}$ and depth $h=4 \mathrm{Km}$ hence $\alpha=a / h \sim 2.5 \times 10^{-4}$. On the other hand the wavelength is $\ell=X_{0}=180 \mathrm{Km}$, hence $\beta=h / \ell \sim 4 K m / 180 K m \sim 2 \times 10^{-2}$. Therefore in this basin of the ocean, the tsunami fits quite well into the regime $\alpha \sim \beta^{2}$ of dispersive nonlinear waves. This is of particular interest because it is this regime of the water waves problem which most clearly supports solutions of the form of coherent dispersive packets.

In contrast, the same considerations in the Andaman Basin give mixed conclusions; we calculate $\alpha=a / h \sim 10^{-3}$ while $\beta=h / \ell \sim 10^{-2}$. This indicates that $\beta^{2} \ll \alpha \ll \beta$, and it is somewhat ambiguous which scaling regime is valid in order to model wave propagation. 
(a)

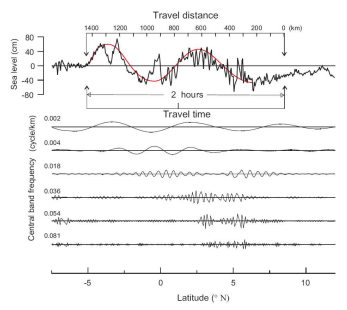

(b)

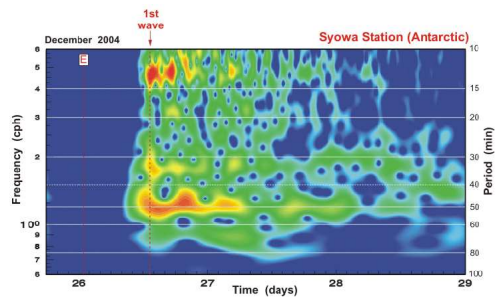

Figure 4. (a) from E. Kulikov, Shirshov Institute of Oceanography, Moscow and Institute of Ocean Sciences, Sydney, BC: 'Dispersion of the Sumatra tsunami waves in the Indian Ocean detected by satellite altimetry', Fisheries and Oceans Canada, Science - Pacific Region website (2005) (b) From the Tsunamis and tsunami research website of the Institute of Ocean Sciences, Sidney BC. This time/frequency plot of the Sumatra tsunami supports the wavepacket hypothesis.

Turning to the data from the tsunami waves in the Indian Ocean taken during the event, we see that indicates that the tsunami wave is experiencing dispersive effects during its propagation. Figure 4(a) represents a trace of the satellite telemetry of wave height during one pass of the Jason 1 Altimetry Satellite (a joint NASA European Space Agency mission, which detected the Indian Ocean tsunami during track 129 of its 10 day/254 track cycle), where it is quite clearly indicated the degree to which low wave number components of the wave form are traveling faster than components with higher wave number. Figure 4(b) gives a Fourier analysis of the data from a wave gage at Syowa Station, Antarctica during the period of several days December 25th - 29th, 2004. The data are presented in a 'wavelet' decomposition, which indicates two strong peak responses during the tsunami period. These are interpreted as the Fourier transform of the main wave packet, centered about the the carrier wavenumber $K_{0}=3 \pi /$ hour of the tsunami event, and its second harmonic $K_{1}=6 \pi /$ hour.

\section{Near shore dynamics}

At the point that tsunami waves arrive at a coastline, the can exhibit very complex near-shore wave dynamics, and there is much that is not well understood. A large wavelength ocean wave incident on a coastline can be dispersed, or its amplitude can be dramatically amplified, depending to a large part on the the width of the continental shelf, the local geometry of the coast, and the angle of incidence of the approaching tsunami. From the accounts of witnesses of the impact of a tsunami, many different things do occur. In some areas affected by the Boxing Day tsunami, the phenomenon was similar to a rising tide, with no breaking 'white water', but being just several times the magnitude of a normal tide, and coming in over a period of twenty minutes instead of six hours. This sort of incidence was also observed during the impact of the 1964 Pacific Ocean tsunami on Vancouver Island, which was generated by the major earthquake in Alaska. More visible, and also more dangerous and dramatic, were the phenomenon of hydraulic jumps, or 'tidal bores' where the mass of water in motion towards the land is rising too fast to do so smoothly, and a jump of water level, of perhaps one or several meters in height is formed. While the wave crests of the tsunami wave packet are traveling at several 


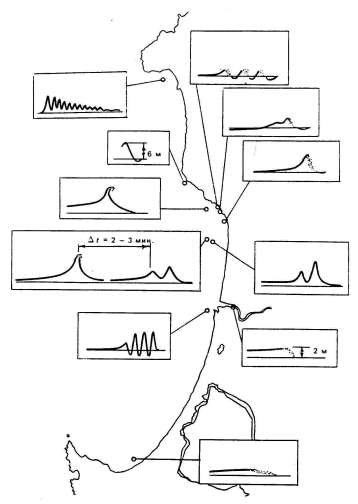

Figure 5. E. Pelinovsky: Dynamics of tsunami waves (1996)[Russian] Nihonkai - Chubu earthquake tsunami on the north Akita coast. N. Shuto, Coastal Eng. Japan (1985), vol. 28 pp. $255-264$

hundred kilometers per hour, the hydraulic jump is much slower, maybe moving at no more that several tens of kilometers per hour. This phenomemon is commonly modeled by a shock wave in the shallow water equations that characterize this near shore regime. This mass of water could possibly recede smoothly, but this is equally or more dangerous, drawing objects and people out to sea.

To illustrate the wide variety of phenomena tha can occur as a tsunami wave is incident on a coastal area, I refer to a diagram compiled by N. Shunto which appears in the book of E. Pelinovsky [12] on the hydrodynamics of tsunami waves. The Nihonkai - Chubu earthquake tsunami occured on May 26, 1983 from a $M_{w} \sim 7.9$ earthquake with epicenter in the Sea of Japan off of the Akita Coast. The event occured at approximately noon, so it happened that there were numerous witnesses on the beaches and shorelines, and a survey of the tsunami waves impact on the North Akita Coast, as reported by many observors, was compiled into the diagram given in Figure 5. It is evident that the phenomena are highly varied, and include hydraulic jumps, series of breaking waves, single large breakers, and others. Another widely circulated image was taken from the air over Kalutara, Sri Lanka during the Boxing Day Tsunami events (Figure 5). One sees large waves moving in a variety of directions through the palms on plots of land ajacent to the beach. We feel that it is a challenging problem to be able to start to quantify the dynamics of large tsunami waves as they impact on coastal areas, for both the purpose of understanding the physics of the phenomenon, as well as to quatify the potential damage of their impact and the possible engineering norms that should be applied to create a tsunami-safe architecture.

\section{Solitary wave interactions}

The second lecture is related to the first in that it is on the topic of nonlinear water waves in the free surface of an ideal fluid. It is in fact a discussion of the classical problem of interactions between solitary water waves. We will restrict our considerations to solitary waves in two dimensions, and to the case in which the bottom boundary of the fluid region is flat. There are a number of reasons why 


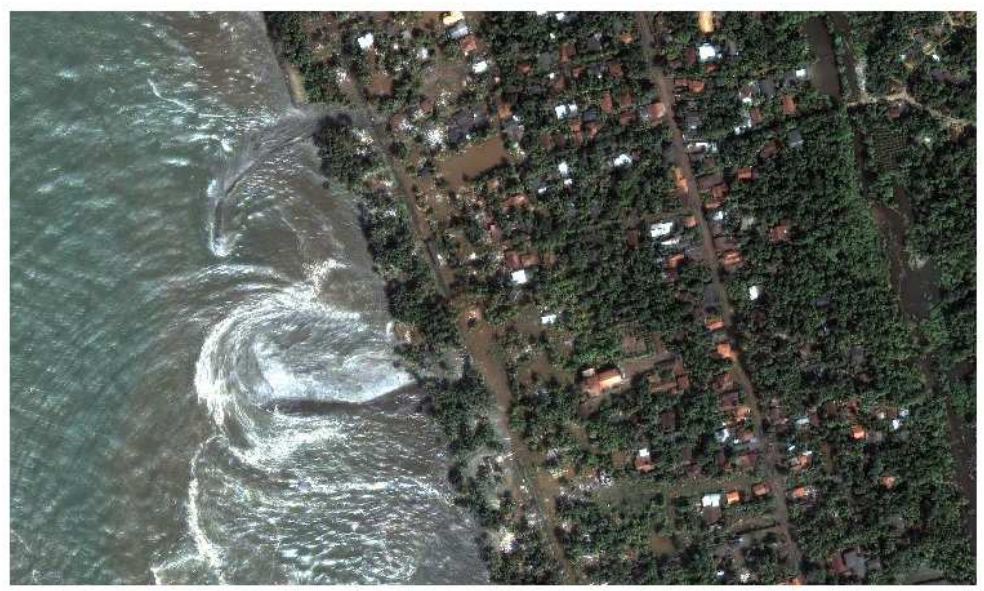

Figure 6. Shoreline of Kalutara, Sri Lanka on 26 December 2004. Photo: DigitalGlobe

this represents an interesting problem. One is that the dynamics of nonlinear waves is an important topic to understand, to further our basic understanding of the oceans next to which we live, and to prepare us for its dangers. A second reason is more mathematical; from the study of the KdV equation we know that from the apropriate point of view it is a completely integrable Hamiltonian system, whose soliton solutions interact with elastic collisions. Since the KdV equation is one of the principal model equations for free surface water waves, it is therefore of interest to understand to what extent its solitary wave solutions deviate from having elastic interactions. There are two basic cases; the interaction between counter-propagating waves (either symmetric collisions or collisions between waves of different amplitudes), and co-propagating or overtaking interactions. This work updates the wellknown numerical simulations of Chen and Street [2], Fenton and Reinecker [8], and Cooker, Weidman and Bale [3]. It has also been compared with the experimental results of Maxworthy [11] and our own experiments [4]. We work with potential flow, for which the velocity potential satisfies (2.1), and the boundary conditions $(2.3)(2.2)$.

\section{Numerical method}

Our numerical method consists essentially in making good approximations for the Dirichlet - Neumann operator (2.6), and using them in a time discretized version of the evolution equations (2.8). This approach was introduced in [7] and used in a variety of settings, including [5][1].

It was already described by J. Hadamard [9] in his Collège de France lectures that Green's function for Laplace's equation is differentiable with respect to the domain on which it is given. Indeed he gave a formula for its variations, and in [10] he proposed hydrodynamical applications. In fact in the appropriate setting it has been shown that the closely related Dirichlet - Neumann operator is analytic with respect to its dependence upon the domain. Putting this into practice in the neighborhood of a fluid domain at rest, we base our simulations on the Taylor expansion of the Dirichlet - Neumann operator to arbitrarily high order in the 
equations of motion (2.8). The first several Taylor approximations to $G(\eta)$ are

$$
\begin{aligned}
G^{(0)} & =D \tanh (h D) \\
G^{(1)} & =D \eta D-G^{(0)} \eta G^{(0)} \\
G^{(2)} & =\frac{1}{2}\left(G^{(0)} D \eta^{2} D-D^{2} \eta^{2} G^{(0)}-2 G^{(0)} \eta G^{(1)}\right) .
\end{aligned}
$$

In our notation, $D=-i \partial_{x}$, and $G^{(0)}$ is a Fourier multiplier operator which is given by the expression

$$
G^{(0)} \xi(x):=\frac{1}{\sqrt{2 \pi}} \int e^{i k x} k \tanh (h k) \hat{\xi}(k) d k
$$

Such expressions can be implemented efficiently using the Fast Fourier Transform. As well, there is a recursion formula for the Taylor series for $G(\eta)$ which can be incorporated into very efficient numerical schemes of arbitrarily high order in the (slope of the) surface elevation $\eta(x)$. This is essentially what we have done in [4] for our study of solitary water wave interactions.

Initial data for our simulations consists of two well separated solitary water waves, of nondimensional amplitudes $S_{1} / h$ and $S_{2} / h$, which are set to collide within the computational domain. The solitary wave profiles for this are generated using the numerical method proposed by M. Tanaka [16], giving us highly accurate results.

\section{Head-on collisions}

This section is concerned with collisions between two counter-propagating solitary waves, of nondimensional elevation $S_{1} / h$ and $S_{2} / h$ respectively. The first simulations presented here are symmetric head-on collisions between two solitary waves of equal amplitudes $S / h$. Features of note are the degree of run-up of the wave crest during the interaction, given by $\sup _{x, t}|\eta(x, t)| / h-2 S / h$; the phase lag due to the moment's hesitation of the crests during their interaction; the change in amplitude of the solitary waves after the interaction, $S / h \mapsto S^{+} / h$; their phase lag $a \mapsto a^{+}$; and the residual waves $\eta_{R}(x, t)$ trailing the solitary waves as they exit the collision. We observe that the solitary waves in head-on collisions always lose a small amount of amplitude due to the collision; $S_{j}^{+}<S_{j}$, although this is very small even for interactions between large solitary waves.

The residual is clearly visible in the figure 8 , but it is essentially too small to be detected in figure 7 without magnification. A snapshot plot of the computation in figure 7 at time $t / \sqrt{h / g}=90$, with magnified vertical scale, is given in the figure 9 .

Viewing the interaction of two solitary waves of amplitude $S / h=0.4$ after long time illustrates the asymptotic tear-shaped form of the residual, as well as the fact that the solitary waves separate from each other and from the the residual after the collision. This is shown in figure 10; it is an indication of the stability of solitary waves to such head-on collisions, at least within this range of amplitudes.

\section{Overtaking collisions}

We have also run simulations of overtaking collisions with this numerical scheme, where we find yet smaller changes of amplitude $\Delta S_{j}:=S_{j}-S_{j}^{+}, j=1,2$ and 


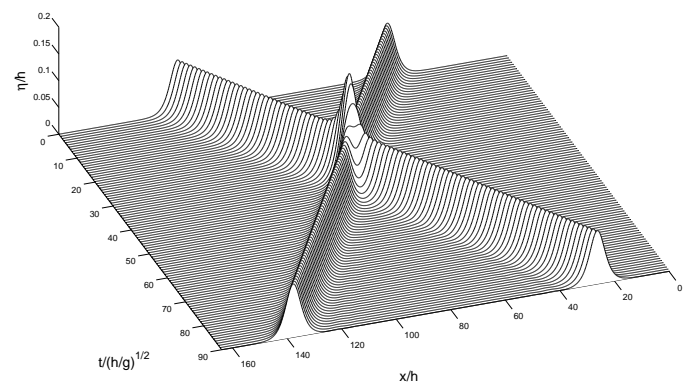

Figure 7. Head-on collision of two solitary waves of equal height $S / h=0.1$ : The amplitude after collision is $S^{+} / h=0.0997$ at $t / \sqrt{h / g}=90$. The phase lag is $\left(a_{j}-a_{j}^{+}\right) / h=0.1370$.

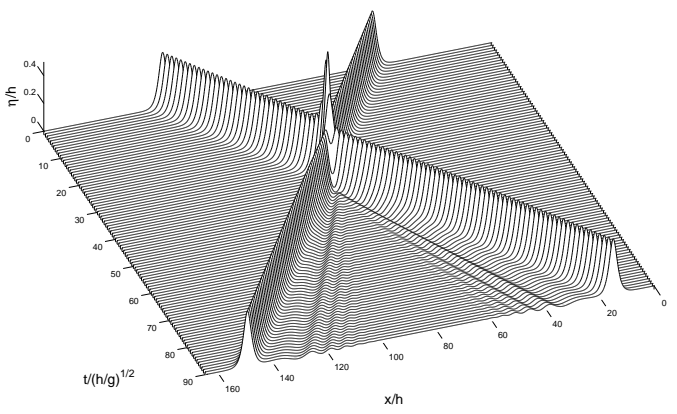

Figure 8. Head-on collision of two solitary waves of equal height $S / h=0.4$ : The amplitude after collision is $S^{+} / h=0.3976$ at $t / \sqrt{h / g}=90$. The phase lag is $\left(a_{j}-a_{j}^{+}\right) / h=0.3257$.

residual $\eta_{R}$ after a collision. Normalize the notation so that the larger incoming solitary wave has amplitude $S_{1}$ and the second $S_{2}$. In these simulations, we observe that the amplitude of the first solitary wave is slightly increased, $S_{1}^{+}>S_{1}$, while the second decreases as is necessary. We also observe that the amplitude of the solution never exceeds the maximum of the amplitudes of the entering and exiting solitary waves, nor at any time does the maximum crest dip below the minimum. Just as in the situation of the two-soliton solution of the KdV equation, the solitary waves experience a positive phase shift due to the collision, as though the two waves were repelling particles. Figure 11 shows the interaction of two solitary waves of amplitudes $S_{1} / h=0.4$ and $S_{2} / h=0.1333$, this being chosen so that the interaction is of the Lax category (b) in its form. The simulation is displayed in a frame of reference in motion at approximately the mean velocity of the two solitary waves.

In figure 12 a view of this simulation with exagerated scale at a time after the interaction shown clearly the very small but nonzero residual. 


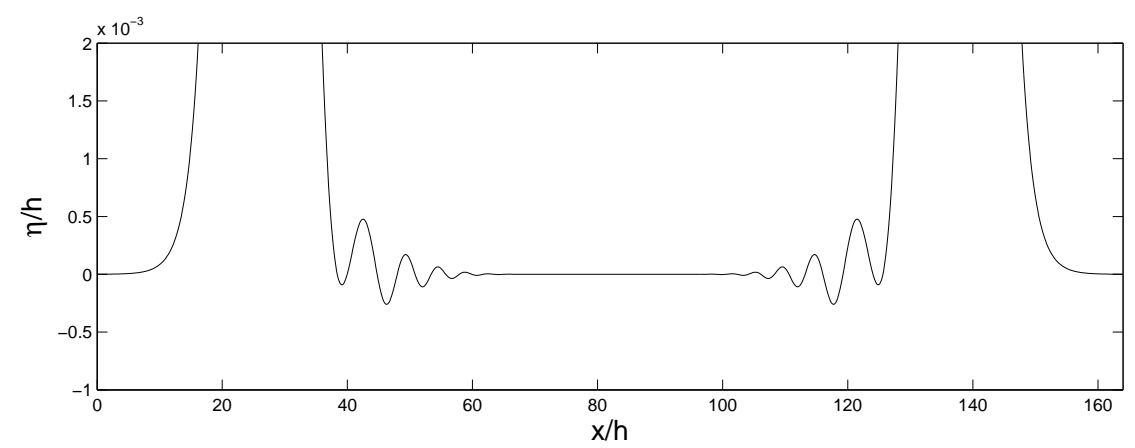

Figure 9. Results of the collision of two solitary waves of equal height $S / h=0.1$ after the collision, at time $t / \sqrt{h / g}=90$. The dispersive residual wave trailing the solitary waves after the collision are visible under magnification.

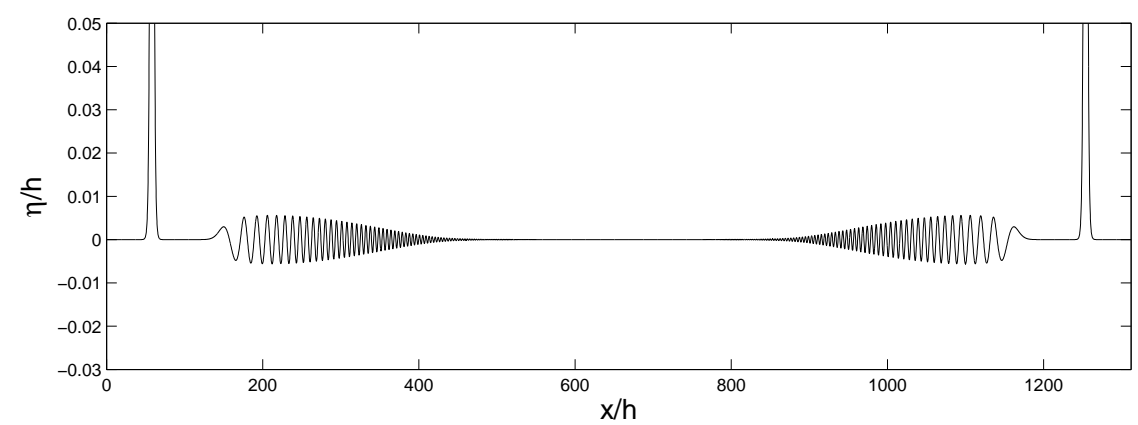

Figure 10. Results of the collision of two solitary waves of equal height $S / h=0.4$ after the collision at time $t / \sqrt{h / g}=780$. The residual has a characteristic tear-shaped envelope

\section{Energy transfer}

Using the conservation laws (2.11)(2.14) and the Hamiltonian (2.5), one can derive a relation between the change in amplitude through a solitary wave interaction and the energy that has been transferred to the residual. Using this, it is also possible to derive a rigorous upper bound on the energy transfer in terms of the parameters $S_{1} / h, S_{2} / h$ of the initial data. The latter analysis appears in [4]. To explain the first relation, an individual solitary wave has mass $M\left(\eta_{S}\right):=m(S)$, momentum $I\left(\eta_{S}, \xi_{S}\right):=\mu(S)$ and energy $H\left(\eta_{S}, \xi_{S}\right):=e(S)$. Our initial data is given by two asymptotically separated solitary waves as $t \mapsto-\infty$, therefore the total mass, momentum and energy of our solution are given by

$$
\begin{aligned}
M_{T} & =m\left(S_{1}\right)+m\left(S_{2}\right) \\
I_{T} & =\mu\left(S_{1}\right)+\mu\left(S_{2}\right) \\
E_{T} & =e\left(S_{1}\right)+e\left(S_{2}\right) .
\end{aligned}
$$

After an interaction has occurred, we will assume that the solution is composed of three distinct components; two solitary waves with possibly different amplitudes $S_{1}^{+}$ and $S_{2}^{+}$, and a residual $\left(\eta_{R}(x, t), \xi_{R}(x, t)\right)$. By conservation, their mass, momenta 


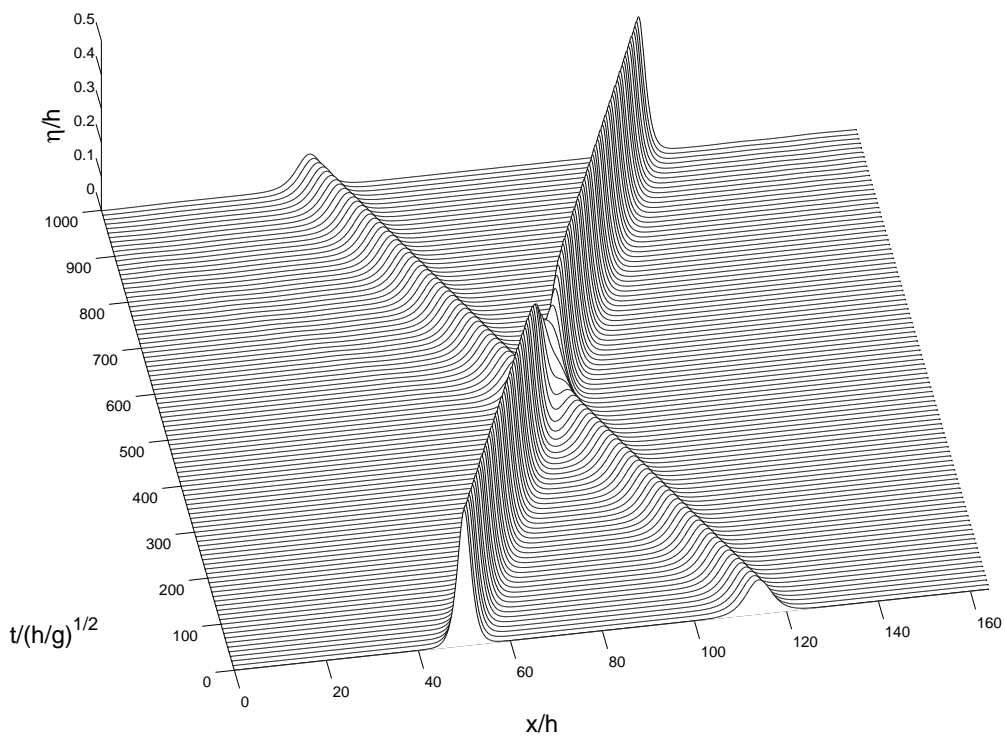

Figure 11. Overtaking collision of two solitary waves of height $S_{1} / h=0.4, S_{2} / h=0.1$ : The amplitudes after collision are $S_{1}^{+} / h=0.4003, S_{2}^{+} / h=0.0999$ at $t / \sqrt{h / g}=1000$ for the large, small wave respectively. The phase shifts are $\left(a_{1}^{+}-a_{1}\right) / h=2.2974$, $\left(a_{2}^{+}-a_{2}\right) / h=3.6159$ respectively.

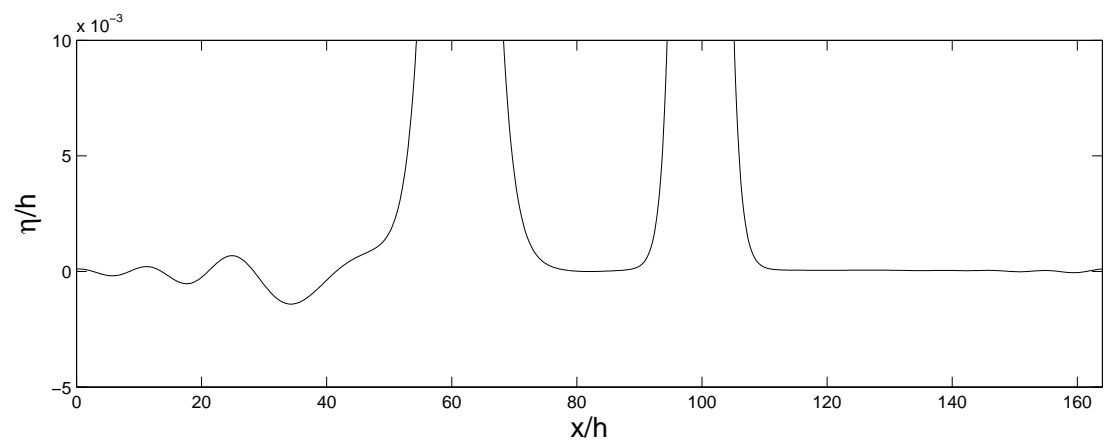

Figure 12. Overtaking collision of two solitary waves of height $S_{1} / h=0.4, S_{2} / h=0.1$ at $t / \sqrt{h / g}=745$, which is after the collision. The vertical scale is magnified in order to observe the dispersive trailing wave generated by the interaction.

and energies satisfy

$$
\begin{aligned}
M_{T} & =m\left(S_{1}^{+}\right)+m\left(S_{2}^{+}\right)+m_{R} \\
I_{T} & =\mu\left(S_{1}^{+}\right)+\mu\left(S_{2}^{+}\right)+\mu_{R} \\
E_{T} & =e\left(S_{1}^{+}\right)+e\left(S_{2}^{+}\right)+e_{R} .
\end{aligned}
$$


Taking the difference, we find that

$$
\begin{aligned}
\left(m\left(S_{1}\right)-m\left(S_{1}^{+}\right)\right)+\left(m\left(S_{2}\right)-m\left(S_{2}^{+}\right)\right) & =m_{R} \\
\left(\mu\left(S_{1}\right)-\mu\left(S_{1}^{+}\right)\right)+\left(\mu\left(S_{2}\right)-\mu\left(S_{2}^{+}\right)\right) & =\mu_{R} \\
\left(e\left(S_{1}\right)-e\left(S_{1}^{+}\right)\right)+\left(e\left(S_{2}\right)-e\left(S_{2}^{+}\right)\right) & =e_{R} .
\end{aligned}
$$

Since the change in amplitude is very small, the difference $m\left(S_{j}\right)-m\left(S_{j}^{+}\right)$is very small, $j=1,2$, and the same for $\mu\left(S_{j}\right)$ and $e\left(S_{j}\right)$. Approximating by the derivative, we conclude that

$$
\begin{aligned}
m_{1}^{\prime} \Delta S_{1}+m_{2}^{\prime} \Delta S_{2} & =m_{R} \\
\mu_{1}^{\prime} \Delta S_{1}+\mu_{2}^{\prime} \Delta S_{2} & =\mu_{R} \\
e_{1}^{\prime} \Delta S_{1}+e_{2}^{\prime} \Delta S_{2} & =e_{R} ;
\end{aligned}
$$

this is now three equations for the two unknowns $\Delta_{j}, j=1,2$, whose solution leads us to an absolute bound on the energy loss due to a collision [4]. Separately from this bound, equations (10.4) specify relationships between the mass, momentum and energy loss to the residual and the change in amplitude $\Delta S_{j}$ from the interaction.

Let us consider the case of symmetric interactions as an example. In this case, $\mu\left(S_{1}\right)=-\mu\left(S_{2}\right)$ and therefore $I_{T}=0$ and $\mu_{R}=0$. The relation (10.4) then reports that

$$
2 m^{\prime}(S)=m_{R}, \quad 2 e^{\prime}(S)=e_{R} .
$$

Since in particular $e(S) \sim S^{3 / 2}$ for small $S$, this predicts that

$$
e_{R} \sim S^{1 / 2} \Delta S .
$$

Figure 13 plots the quantity $e(S)$ for a range of simulations ranging from $S=0.025$ to $S=0.5$, verifying its power law behavior. Figure 14 gives our measured values of $\Delta S$ for these simulations, while figure 15 gives the energy $e_{R}$ of the residual. The adherence of thie data to the asymptotic relation (10.6) is quite convincing. The deviation of the lowest two data points from the power law behavior is due to the long relaxation time of small solutions to their asymptotic values after an interaction, we believe.

Our data, particularly in figure 14, are at odds with the asymptotic predictions of C.-H. Su and R. M. Mirie [15], who put forward that $\eta_{R}=\mathcal{O}\left(S^{3}\right)$ while $\Delta S=o\left(S^{3}\right)$.

The relations (10.4) give a nontrivial result on the mass and energy of the residual and the quantities $\Delta S_{j}^{+}$in the case of overtaking collisions. From our simulations and from those in [8], it is observed that $\Delta S_{1}<0$. That is, the larger overtaking solitary wave gains a (small amount of) amplitude due to the collision, at the expense of the smaller one. We note that $e(S)$ is a convex function of $S$, at least over the range of values of $S$ being considered. The implication of (10.4) is that, since $e_{R} \geq 0$, it must be that $\left|\Delta S_{1}\right|<\Delta S_{2}$; the larger solitary wave cannot gain more amplitude than the smaller one loses. Using this, a related argument applied to the relation for mass implies that $m_{R}<0$, because in this case $m^{\prime}(S)$ is decreasing in $S$. This fact is seen in the slight depression left behind two separating solitary waves after their interaction.

Acknowledgements: This research has been supported in part by the Canada Research Chairs Program, the NSERC under operating grant \#238452, and the NSF under grant \#DMS-0070218. All of the numerical simulations in this note were performed by P. Guyenne. 


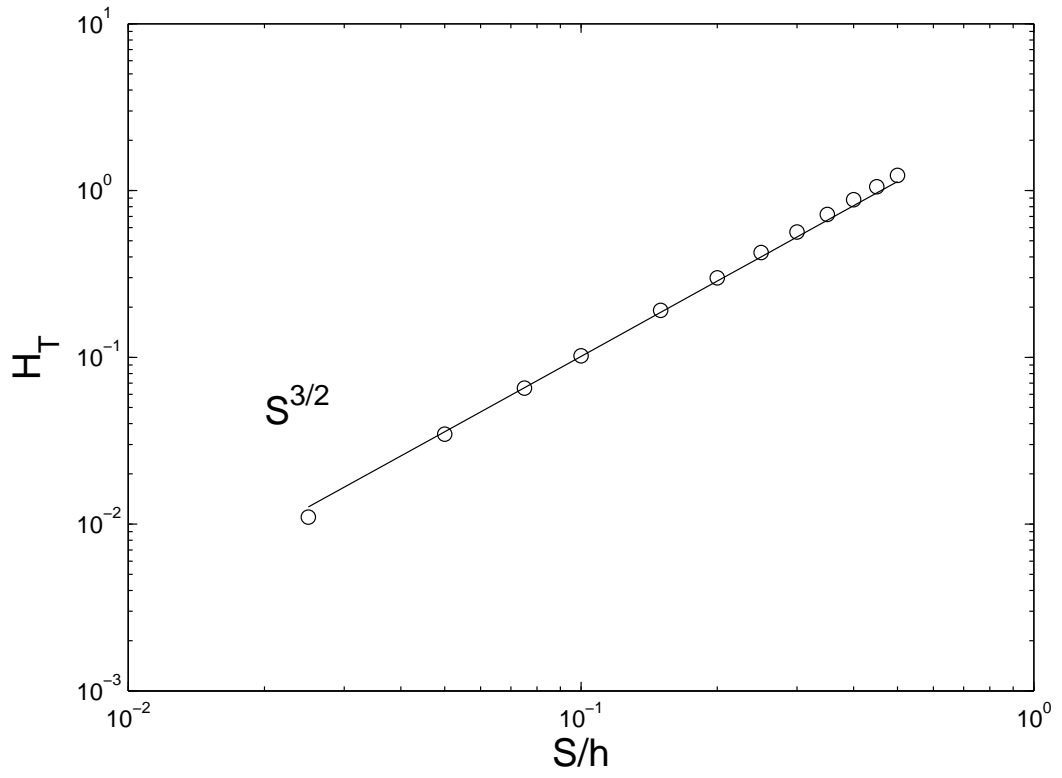

Figure 13. Total energy $E_{T}$ vs. wave amplitude $S / h$ : numerical results (circles), power law $(S / h)^{3 / 2}$ (solid line).

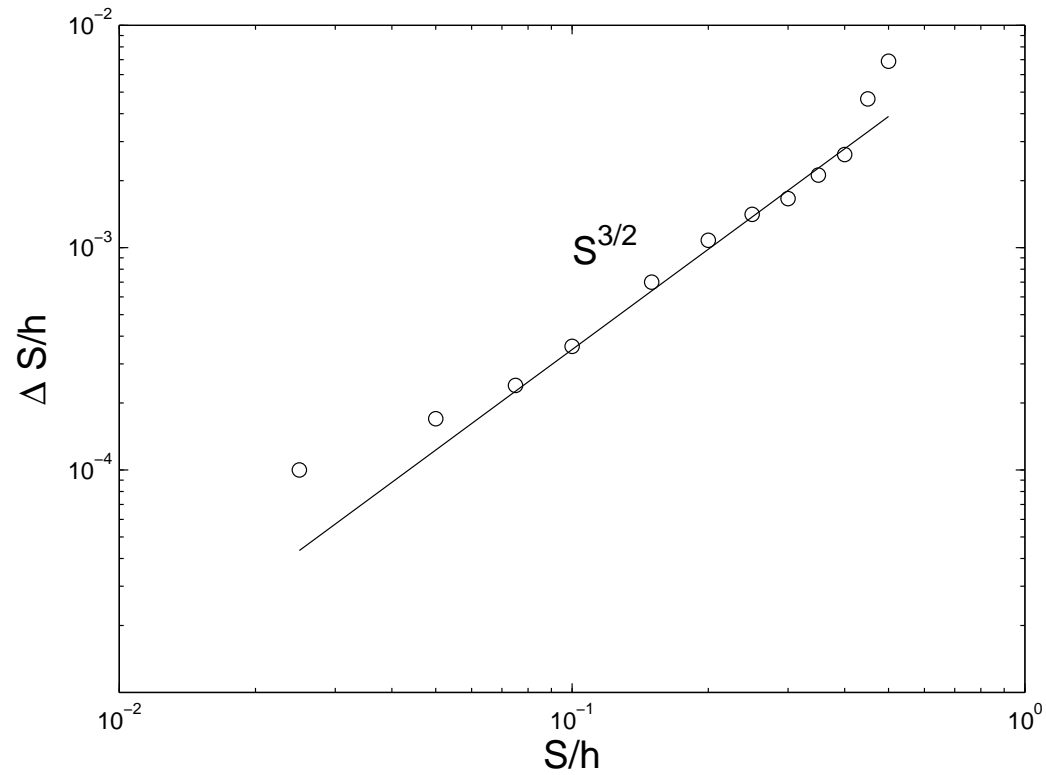

Figure 14. Change in amplitude $\Delta S / h=\left(S-S^{+}\right) / h$ vs. wave amplitude $S / h$ : numerical results (circles), power law $(S / h)^{3 / 2}$ (solid line).

\section{References}

[1] W. J. D. Bateman, C. Swan and P. Taylor. On the efficient numerical simulation of directionally spread surface water waves. J. Comput. Phys. 174, 277 (2001). 


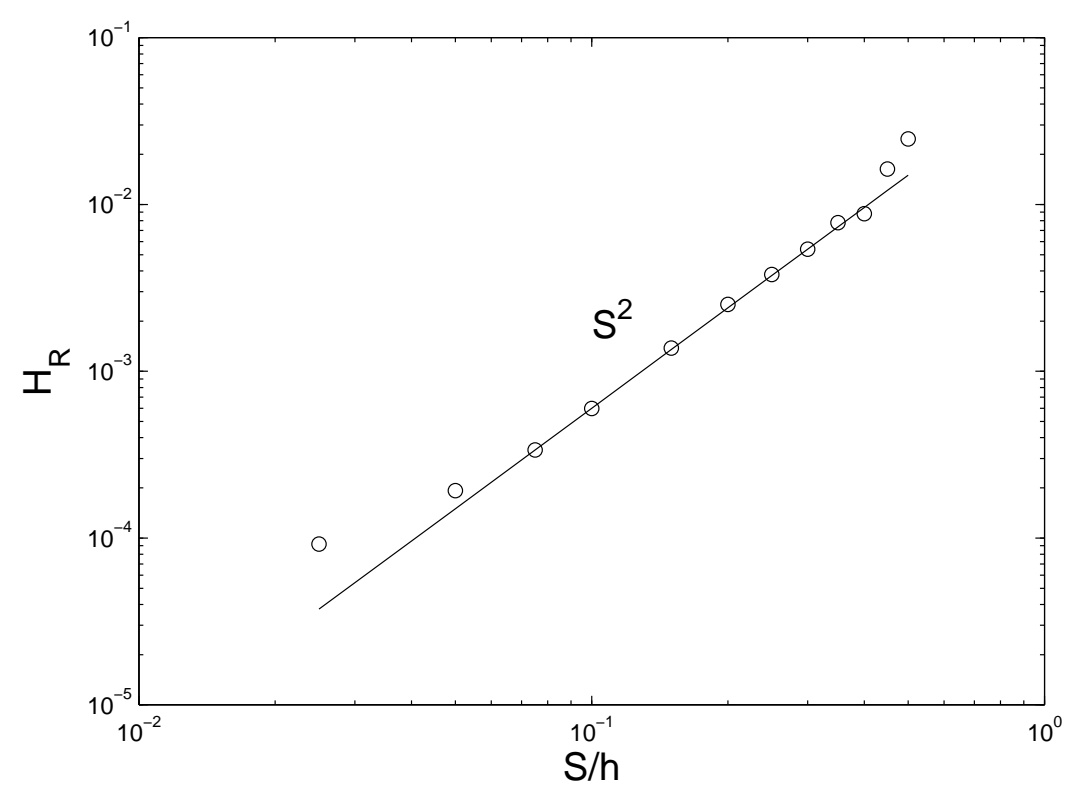

Figure 15. Energy of the residual $e_{R}$ vs. nondimensional wave amplitude $S / h$ : numerical results (circles), power law $(S / h)^{2}$ (solid line).

[2] R. K.-C. Chan, and R. Street. A computer study of finite amplitude water waves. J. Comput. Phys. 6, 68 (1970).

[3] M. J. Cooker, P. D. Weidman, and D. S. Bale. Reflection of a high-amplitude solitary wave at a vertical wall. J. Fluid Mech. 342, 141 (1997).

[4] W. Craig, P. Guyenne, J. Hammack, D. Henderson and C. Sulem. Solitary wave interactions. Physics of Fluids (2006), to appear.

[5] W. Craig \& D. Nicholls. Traveling two and three dimensional capillary gravity waves. SIAM: Math. Analysis 32 (2000), pp. 323-359.

[6] W. Craig, P. Guyenne, D. Nicholls and C. Sulem. Hamiltonian long wave expansions for water waves over a rough bottom," Proc. R. Soc. A 461, 839 (2005).

[7] W. Craig and C. Sulem. Numerical simulation of gravity waves. J. Comp. Phys.108, pp. 73-83, (1993).

[8] J. D. Fenton, and M. M. Rienecker. A Fourier method for solving nonlinear water-wave problems: application to solitary-wave interactions. J. Fluid Mech. 118, 411 (1982).

[9] J. Hadamard. Leçons sur le calcul des variations. Hermann, Paris, 1910; paragraph $\S 249$.

[10] J. Hadamard. Sur les ondes liquides. Rend. Acad. Lincei 5, (1916) no. 25, pp. 716-719.

[11] T. Maxworthy. Experiments on the collision between two solitary waves. J. Fluid Mech. 76, 177 (1976).

[12] E. Pelinovsky. Hydrodynamics of tsunami waves (Russian) Russian Academy of Sciences (1996).

[13] R. Rosales and G. Papanicolaou. Gravity waves in a channel with a rough bottom. Stud. Appl. Math. 68, (1983) pp. 89-102.

[14] G. Schneider and C. E. Wayne. The long-wave limit for the water wave problem. I. The case of zero surface tension, Comm. Pure Appl. Math. 53, 1475 (2000).

[15] C. H. Su, and R. M. Mirie. On head-on collisions between two solitary waves. J. Fluid Mech. 98, 509 (1980). 
[16] M. Tanaka. The stability of solitary waves. Phys. Fluids 29, 650 (1986).

[17] V. Titov, A. B. Rabinovich, H. O. Mofjeld, R. E. Thompson and F. I. Gon'alez. The global reach of the 26 December Sumatra tsunami. Science 309 no. 5743, Sept. 232005 , pp. 2045-2048.

[18] J. D. Wright. Corrections to the KdV approximation for water waves. SIAM J. Math. Anal. 37, 1161 (2005).

[19] V.E. Zakharov. Stability of periodic waves of finite amplitude on the surface of a deep fluid. J. Appl. Mech. Tech. Phys. 9 (1968), pp. 190-194. 